\title{
Changes in Impact Signals and Muscle Activity in Response to Different Shoe and Landing Conditions
}

\author{
by \\ Xi Wang ${ }^{1,2}$, Shen Zhang ${ }^{1}$, Weijie Fu ${ }^{1}$
}

Few rigorous scientific studies have investigated how the corresponding neuromuscular activity in the lower extremity occurs during different landing control movements in response to different impact signals. This study aimed to determine the potential shoe effects on impact signals, neuromuscular responses and their possible interactions in different human landing movements. Twelve male basketball players were required to wear high-cushioned basketball shoes (BS) and minimally cushioned control shoes (CC) to perform active drop jump landings (DJL) and passive landings (PL). Ground reaction forces and EMG amplitude (root mean square, EMGRMS) of the leg muscles within 50 $m s$ before and after the landing movements were collected simultaneously. No shoe effect was found on the characteristics of impact signals and neuromuscular activity during the contact phase of DJL. By contrast, for PL, the values of maximal ground reaction force and the peak loading rate were evidently lower in the BS condition than in the CC condition $(p<0.05)$. Meanwhile, the EMGRMS of all muscles demonstrated a significant decrease in the BS condition compared with the CC condition within $50 \mathrm{~ms}$ after contact $(p<0.05)$. These findings suggest that under the condition in which related muscles are activated improperly, a neuromuscular adaptation occurs in response to different impact signals.

Key words: vertical ground reaction force; EMG amplitude; drop landing.

\section{Introduction}

During two-footed landings from vertical jumps, the peak magnitude of vertical reaction forces has been found to range from 3.5 to 6 times BW (Gross and Nelson, 1988). Previous investigations reported a close relationship between the great shock in strenuous landings and lower-limb injuries, that is, repetitive excessive loading can induce acute injuries (Beynnon et al., 2005; McNitt-Gray, 1993) and overuse damages (Agel et al., 2007; Borowski et al., 2008). Thus, to prevent sports injuries in athletic activities, footwear manufacturers have been focusing on designing shoes that can attenuate a shock wave, and thus the concept of cushioning has been widely used since the 1970s (Clarke et al., 1983).
Current investigations into impact forces have focused not only on the magnitude, timing and the loading rate, but also on the reactions and muscular responses of the musculoskeletal system (Brüggemann et al., 2011). A series of concepts about the effects of impacts has been provided during the past ten years (Boyer and Nigg, 2007; Nigg and Wakeling, 2001). Impacts are regarded as input signals into the human locomotor system, which produce a shock wave and meantime initiate the vibrations of lower extremity soft tissues. These signals are sensed and the central nervous system responds by adjusting, if necessary, the activation of the relevant major muscle groups (Nigg and Wakeling, 2001). Basically, the musculoskeletal system responds to

1 - Key Laboratory of Exercise and Health Sciences of Ministry of Education, Shanghai University of Sport, Shanghai, China.

2 - School of Kinesiology, Shanghai University of Sport, Shanghai, China. 
this input signal by changing the activation levels of muscles to avoid a resonance situation. These neuromuscular changes are supposed to reduce vibrations and thus avoid lower extremity injuries (Boyer and Nigg, 2006).

Generally, impact force substantially varies for different landing speeds (Frederick and Hagy, 1986; Hamill et al., 1983), different lower limb postures (Derrick et al., 2002), and/or the hardness (or material) of the shoe midsole (Clarke et al., 1983). Theoretically, different shoe conditions potentially provide a specific impact input into the musculoskeletal system. One relevant study by Boyer and Nigg (2004) indicated that within a given touchdown speed, shoe midsole material changed both the loading rate and the frequency of impact force. Specifically, a change in the properties of the midsole had a significant effect on both the loading rate and magnitude of the impact peak, especially at higher landing speeds. Meanwhile, reduced impact loading and longer times to impact peak force were also achieved with softer midsole materials (Lafortune et al., 1996). However, a respectable sum of results (Clarke et al., 1983; Nigg et al., 1987) observed during impact-related landings with footwear incorporating midsoles of different hardness still conflicted with the aforementioned positive findings. A well-known study by Nigg et al. (1987) reported no significant effect of midsole material when attempting to influence the peak magnitude or the loading rate. The lack of consistent results with respect to shoe/surface effects on impact forces in those studies has been attributed to muscle adaptations in the lower extremity, such as changes in the initial foot and leg angle, touch-down velocity and leg stiffness (Gerritsen et al., 1995).

Recently, surface electromyography (EMG) has been widely applied to investigate and evaluate muscle activity/adaptation (Beck et al., 2012; Camic et al., 2013; Gabriel et al., 2007; Rocha-Júnior et al., 2015). The time domains of the EMG signal reflect the changes in electrical activity and motor unit recruitment during muscle contraction, which are generally regarded to be sensitive for investigating the changes in motor control patterns in movement tasks and interface/shoe configurations (Basmajian and DeLuca, 1985). Boy and Nigg (2007) reported that the shoe condition produces significant differences in EMG intensity of leg muscles for the post-impact window, but not for the pre-impact period during a pendulum impact experiment. For shoe cushioning, studies have similarly indicated that lower limb muscle activity can be "tuned" with shoes having different midsole materials/hardness to accommodate the impact force at heel strike (Wakeling et al., 2002). However, most of the work that focused on the role of the shoe on impact signals, and subsequently on neuromuscular responses, was conducted during running movements (Boyer and Nigg, 2004; Boyer and Nigg, 2007; Wakeling et al., 2002) and not during strenuous landing maneuvers. Furthermore, comprehending how the corresponding neuromuscular activity occurs during different landing control movements in response to different impact signals will be helpful to better understand the changes in the motor pattern and neuromuscular control in the lower extremity.

Based on the above observation, the current study aimed to determine the shoe effects on impact signals, neuromuscular responses and their possible interactions during active (drop jump landing, DJL) and passive landings (PL) from different drop heights. Firstly, it was hypothesized that shoe intervention would not significantly influence the peak impact force, the peak loading rate, as well as EMG amplitude during active landings; secondly, that wearing cushioned shoes would significantly reduce impact forces and activation levels of the leg muscles during passive landings.

\section{Material and Methods}

\section{Participants}

Twelve male basketball players (age: $23.7 \pm$ 2.7 years, body height: $178.3 \pm 2.5 \mathrm{~cm}$, body mass: $70.1 \pm 4.6 \mathrm{~kg}$ ) were recruited for this experiment. The inclusion criteria for the participants were (1) more than five year experience of basketball training, and (2) absence of musculoskeletal injuries of the lower extremity six months prior to testing. Potential participants with a history of significant foot or lower-limb problems or systemic or neurological disorders were excluded from the study. A power analysis showed that the sample size was sufficient to provide more than $80 \%$ statistical power in the experimental design. Each participant signed an informed consent form 
approved by the Ethics Committee of the Shanghai University of Sport before the commencement of the study.

\section{Experimental procedure}

Landing measurement consisted of an active landing (drop jump landing, DJL) and a passive landing (PL) from heights of 30,45 and $60 \mathrm{~cm}$. The landing conditions will henceforth be referred to as DJL30 (PL30), DJL45 (PL45) and DJL60 (PL60), respectively. The order in which DJL and $\mathrm{PL}$, as well as the drop heights, were executed was random. For each participant, three successful trials at each landing height were selected for analysis.

The DJL technique required the participants to 1) drop off from the elevated platform down onto the force plate, and then 2) immediately jump vertically up off the ground. No uniform jump height was required in this study. The average jump height in DJL was $38.5 \pm 4.7 \mathrm{~cm}$, with no significant effect of the shoe observed. For the PL task, the participants were instructed to stand stably on the elevated platform. The base of the platform was then dropped by manually removing a bolt, which would cause a sudden drop with unpredictable timing. The participants then fell down onto the force plate without warning. Our idea was to examine the cushioning effect in an activity that was well controlled kinematically in advance of the first impact (DJL), and then in the same condition with less control in advance of the landing (PL). The objective of the DJL was to land and take-off, whereas that of the PL was to land stably. These two landing activities are different maneuvers that involve different landing techniques. For each participant, all 36 trials were completed within two hours. A rest period of 1-2 min was provided between trials to ensure that participants did not get fatigued over the duration of the experiment.

\section{Measures}

$3 D$ force plate

Two $90 \times 60 \mathrm{~cm}$ three-dimensional force plates (9287B, Kistler Corporation, Switzerland), embedded into the floor, were utilized to collect vertical ground reaction force (GRF) data. The sampling rate was set at $1200 \mathrm{~Hz}$.

Surface electromyography

A Biovision system (Biovision, Wehrheim, Germany) was used to record the surface EMG signals from the rectus femoris (RF), biceps femoris (BF), tibialis anterior (TA) and lateral gastrocnemius (LG) muscles in the dominant leg. Its amplifier's common mode rejection ratio (CMRR) was $120 \mathrm{~dB}$ with a signal-to-noise ratio (SNR) greater than $50 \mathrm{~dB}$. Disposable bipolar $\mathrm{Ag} / \mathrm{AgCl}$ surface electrodes were placed on the referenced positions of these muscles (Fu et al., 2012; Huang et al., 2005). The EMG signals and force data were stored simultaneously at a sampling rate of $1200 \mathrm{~Hz}$ with the data acquisition system and DASYLab software (8.0, DATALOG $\mathrm{GmbH}$, Moenchengladbach, Germany). The leg considered dominant was the preferred one to kick a ball (Lawrence et al., 2008). Regarding leg lateralization, this study used the peak vertical ground reaction force (Fzmax) and the occurrence time of $F$ zmax ( $\left.t_{-} F_{\text {peak }}\right)$ to verify the statistical differences between the dominant and nondominant legs of the tested subjects during bipedal landings (DJL and PL) from heights of 30, 45 and $60 \mathrm{~cm}$. Paired sample $t$-test results showed no significant differences in terms of $F_{p e a k}$ and t_Fpeak between the limbs in either landing condition. These findings are in accordance with the results of Wikstrom et al. (2006), who revealed the absence of bilateral deficits in FZmax and t_F Feak. Therefore, we chose the dominant leg, i.e., the preferred leg used to kick a ball (Lawrence et al., 2008), to record and analyze the EMG.

Testing shoes

A landing style was a factor to be taken into account in choosing the experimental shoes. In addition to the observation that the participants landed on the forefoot from drop jump landings and passive landings, we noted that the heels might also contact the ground during the impact phase of landings. This could be partially or fully. Therefore, two shoe conditions that exhibited different cushioning attributes both in the forefoot and heel regions were used in this study. One type of the shoe was a basketball shoe (BS) with a maximized cushioning phylon midsole (viscoelastic) and a full-length cushioning unit in both the forefoot and heel regions. Specifically, the midsole was made of $8-10 \mathrm{~mm}$ thick, low-hardness ethylene vinyl acetate (EVA) foam. The other shoe was a minimally cushioned shoe (control condition, CC) consisting of a rubber outsole $(4-6 \mathrm{~mm})$ and a thin foam insole but without a midsole. The latter was used to simulate the barefoot condition without leaving the foot 
completely unprotected. The order of the shoe conditions was randomized.

\section{Data analysis}

Impact signal

The main variables discussed in this study for impacts were the peak vertical GRF (Fzmax) normalized to body mass, the time to Fzmax and the peak loading rate (Gzmax) normalized to body mass.

Muscle activity

The EMG data were analyzed using DASYLab software. The raw signals were bandpass filtered at $10-500 \mathrm{~Hz}$, and then full-wave rectified ( $\mathrm{Fu}$ et al., 2014). The EMG amplitudes were normalized as a percentage of the highest value recorded during the 18 trials of drop jump landings (Ruan and Li, 2010). The EMG signal was normalized using a method that divided each point constituting the EMG process by using the peak value acquired from the same EMG during the drop jump maneuvers (Horita et al., 2002). The root mean square of the muscle activity (EMGRMs) was calculated during the pre- and post-activation phases of the landing with the following equations:

$$
R M S=\sqrt{\frac{1}{T} \int_{t}^{t+T} E M G^{2}(t) \cdot d t}
$$

Where $t$ is the onset of signal and $T$ is the time interval of each phase, $X_{n}$ is a set of consecutive EMG signals. Specifically, this study defined the phases as follows: the pre-activation phase occurring $50 \mathrm{~ms}$ before ground contact ( -50 $\mathrm{ms}$ ) and the post-activation phase occurring $50 \mathrm{~ms}$ after the touchdown (+50 ms).

\section{Statistics}

The distribution of all dependent variables was examined using the Shapiro-Wilk test and was found not to differ significantly from normality. A two-way repeated measures analysis of variance (shoe $\times$ height) was utilized to explore the differences between the conditions and the interaction effect. Tukey's post hoc tests were executed to determine the individual significant differences using SPSS 13.0. The level of significance was set at $\alpha=0.05$.

\section{Results}

\section{Impact signal}

Peak vertical GRF

There was no significant shoe $\times$ height interaction on the peak vertical GRF. During DJL, no significant difference was found between the two shoe conditions in FZmax (Table 1 and Figure 1). Meanwhile, the time to Fzmax was also found to have no significant difference between the two shoes. By contrast, for PL, the peak vertical force while wearing basketball shoes was significantly lower than that of the control shoes at all drop heights $(p<0.05)$ (Table 1 and Figure 1$)$. As expected, FZmax increased with the drop heights increasing from 30 to $45 \mathrm{~cm}$ and from 45 to $60 \mathrm{~cm}$ in both landing styles (Table 1).

Peak loading rate

No significant interaction was found for the peak loading rate between shoe condition and drop height. During the contact phase of DJL, the patterns of the loading rate curves under the BS and CC conditions were similar. By contrast, for PL, the effect of basketball shoes on impacts significantly decreased the peak loading rate (Table 1). Specifically, the ANOVA results indicated the lack of any major effect of the shoe type for Gzmax in the DJL task at three heights. However, the post hoc comparisons demonstrated that Gzmax in the BS condition was significantly lower than that in the $\mathrm{CC}$ condition at three landing heights in the PL task (Table 1). In addition, the amplitude of GZmax in both DJL and PL was sensitive to the changes of heights as expected (Table 1).

\section{EMG amplitude}

Pre-activation phase (-50 ms)

There was no significant shoe $\times$ height interaction in the pre-activation of the normalized EMG amplitude (EMGRMs). For the four muscles tested (RF, BF, TA and LG), no significant shoe effect was found in the pre-activation of EMGRMS in either DJL or PL at all three landing heights (Figure 2 and Figure 3a). The EMG intensity of the lateral gastrocnemius ( $p=0.048$ ) was significantly reduced only at the $60 \mathrm{~cm}$ height of DJL under the BS condition. Thus, drop height proved to be a factor which significantly changed the values of pre-activation of all muscles in DJL, except for LG (Table 2).

Post-activation phase (+50 ms)

No significant interaction was found for postactivation of the EMG amplitude between the shoe type and drop height. On average, no significant differences in EMGRMs were observed for any of the tested muscles during the post- 
activation phase of DJL (Figure 3b). However, for $\mathrm{PL}$, the shoe factor proved to be the most relevant factor $(p<0.05)$ to the changes in the EMG amplitude variable (Table 2). Specifically, the EMGrms of RF, BF, TA and LG indicated a significant decrease in the BS condition compared with the CC condition from at least one drop height $(p<0.05)$ (Figure $3 b)$. Furthermore, the post hoc comparisons demonstrated that postactivation of EMGrms in the BS condition was significantly lower than that in the CC condition for TA at all landing heights, for LG at PL30 and PL45, for RF at PL60, and for BF at PL45 and PL60, respectively (Figure 3b).

Table 1

The effect of shoe condition on the peak impact (F $\left.F_{\max }\right)$ and peak loading rate $\left(G_{Z \max }\right)$ in different landing tasks.

\begin{tabular}{|c|c|c|c|c|c|c|c|}
\hline \multirow{2}{*}{$\begin{array}{l}\text { Landing } \\
\text { style }\end{array}$} & \multirow{2}{*}{$\begin{array}{l}\text { Shoe } \\
\text { group }\end{array}$} & \multicolumn{3}{|c|}{$\mathrm{FZZmax}_{\text {max }}(\mathrm{BW}$} & \multicolumn{3}{|c|}{$\mathrm{GZmax}_{\text {mW/s }}(\mathrm{BW}$} \\
\hline & & $30 \mathrm{~cm}^{t \ddagger}$ & $45 \mathrm{~cm}^{\ddagger}$ & $60 \mathrm{~cm}$ & $30 \mathrm{~cm}^{+\ddagger}$ & $45 \mathrm{~cm}^{\ddagger}$ & $60 \mathrm{~cm}$ \\
\hline \multirow{4}{*}{$\begin{array}{l}\text { Drop } \\
\text { jump } \\
\text { landing }\end{array}$} & BS & $2.13 \pm 0.51$ & $2.74 \pm 0.42$ & $3.59 \pm 0.81$ & $120.8 \pm 18.2$ & $208.5 \pm 40.1$ & $251.2 \pm 61.5$ \\
\hline & $\mathrm{CC}$ & $2.17 \pm 0.50$ & $2.82 \pm 0.80$ & $3.60 \pm 0.64$ & $128.5 \pm 32.8$ & $228.7 \pm 52.3$ & $295.6 \pm 66.9$ \\
\hline & Diff.\% & $-1.9 \%$ & $-2.7 \%$ & $-0.4 \%$ & $-5.6 \%$ & $-8.9 \%$ & $-14.7 \%$ \\
\hline & $p$ & 0.857 & 0.884 & 0.948 & 0.724 & 0.597 & 0.151 \\
\hline \multirow{4}{*}{$\begin{array}{l}\text { Passive } \\
\text { landing }\end{array}$} & BS & $3.29 \pm 0.47$ & $3.56 \pm 0.80$ & $4.06 \pm 0.71$ & $262.5 \pm 47.8$ & $318.2 \pm 67.3$ & $340.9 \pm 84.6$ \\
\hline & $\mathrm{CC}$ & $3.90 \pm 1.16$ & $4.35 \pm 1.02$ & $4.73 \pm 0.84$ & $349.4 \pm 63.7$ & $398.3 \pm 83.3$ & $438.6 \pm 77.5$ \\
\hline & Diff.\% & $-16.1 \%$ & $-18.3 \%$ & $-14.3 \%$ & $-25.1 \%$ & $-21.2 \%$ & $-22.4 \%$ \\
\hline & $p$ & $0.046^{*}$ & $0.043^{*}$ & $0.047^{*}$ & $0.029 *$ & $0.036^{*}$ & $0.032^{*}$ \\
\hline
\end{tabular}

BS, basketball shoe; CC, control condition. Diff.\% - percentage difference between the BS and the CC divided by the data of CC.

* Significantly different between shoes in the same landing height with $p<0.05$.

+ Significantly different from $45 \mathrm{~cm}$ in the same landing task with $p<0.05$.

$\ddagger$ Significantly different from $60 \mathrm{~cm}$ in the same landing task with $p<0.05$.

\begin{tabular}{|c|c|c|c|c|c|c|c|}
\hline \multicolumn{8}{|c|}{$\begin{array}{c}\text { Effects of the shoe type factor and the drop height factor (p-values) on muscle activation } \\
\left(E M G_{R M S}\right) \text { of the rectus femoris }(R F) \text {, biceps femoris }(B F) \text {, tibialis anterior }(T A) \text { and lateral } \\
\text { gastrocnemius (LG) in different landing tasks. }\end{array}$} \\
\hline \multirow{2}{*}{ Phase } & \multirow{2}{*}{$\begin{array}{l}\text { Muscle } \\
\text { group }\end{array}$} & \multicolumn{3}{|c|}{ Drop jump landing } & \multicolumn{3}{|c|}{ Passive landing } \\
\hline & & Shoe & Height & Interaction & Shoe & Height & Interaction \\
\hline \multirow{4}{*}{$\begin{array}{c}\text { Pre- } \\
\text { activation }\end{array}$} & RF & 0.625 & $0.017^{*}$ & 0.392 & 0.542 & 0.184 & 0.284 \\
\hline & BF & 0.839 & $0.026^{*}$ & 0.420 & 0.728 & $0.037^{*}$ & 0.583 \\
\hline & TA & 0.413 & $0.015^{*}$ & 0.719 & 0.480 & 0.382 & 0.662 \\
\hline & LG & 0.156 & 0.273 & 0.831 & 0.433 & 0.079 & 0.417 \\
\hline \multirow{4}{*}{$\begin{array}{c}\text { Post- } \\
\text { activation }\end{array}$} & RF & 0.452 & 0.621 & 0.738 & 0.152 & 0.196 & 0.633 \\
\hline & $\mathrm{BF}$ & 0.575 & 0.382 & 0.439 & $0.042^{*}$ & 0.272 & 0.582 \\
\hline & TA & 0.328 & 0.422 & 0.192 & $0.010^{*}$ & 0.843 & 0.665 \\
\hline & LG & 0.466 & 0.821 & 0.529 & $0.034^{*}$ & 0.475 & 0.286 \\
\hline \multicolumn{8}{|c|}{ * Significant $p(p<0.05)$} \\
\hline
\end{tabular}


Drop drop landing
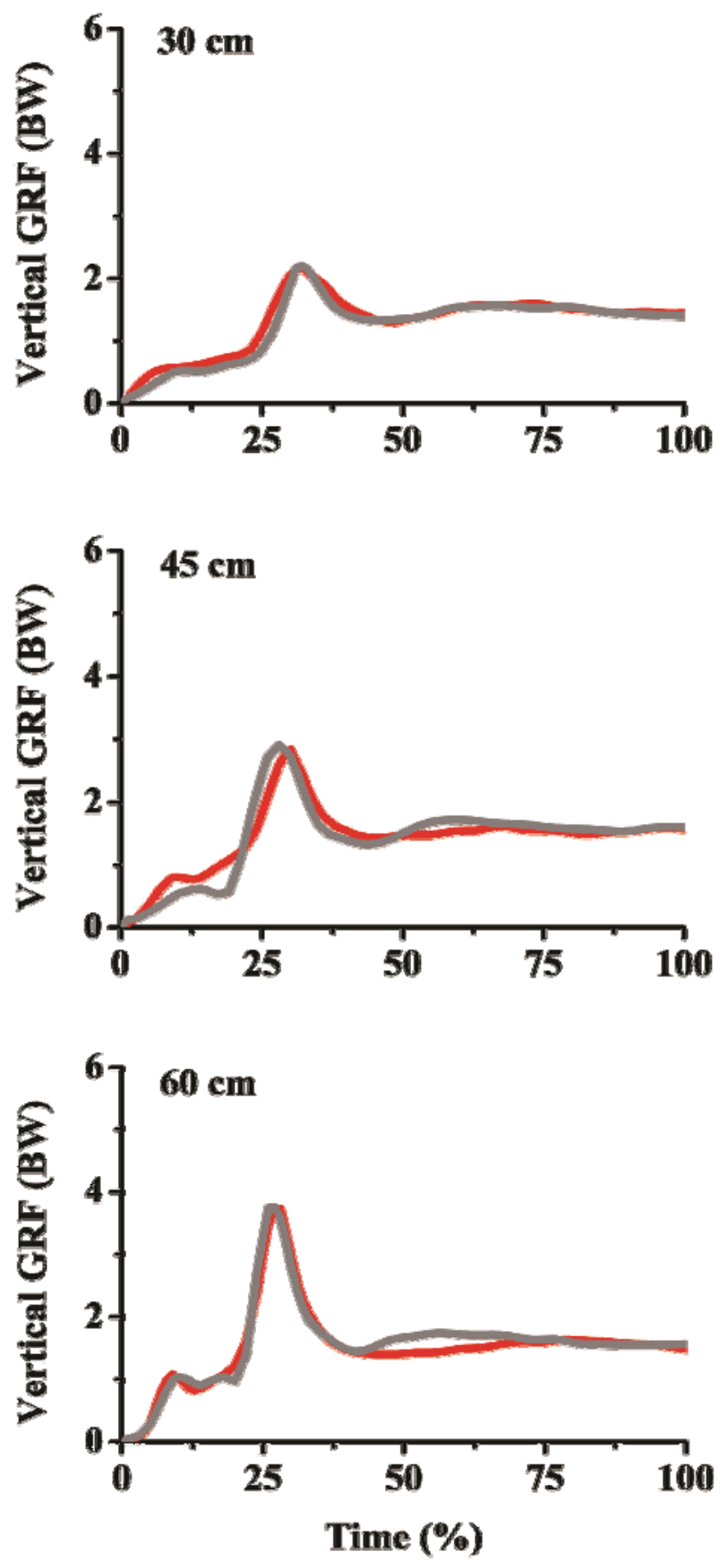

Passive landing
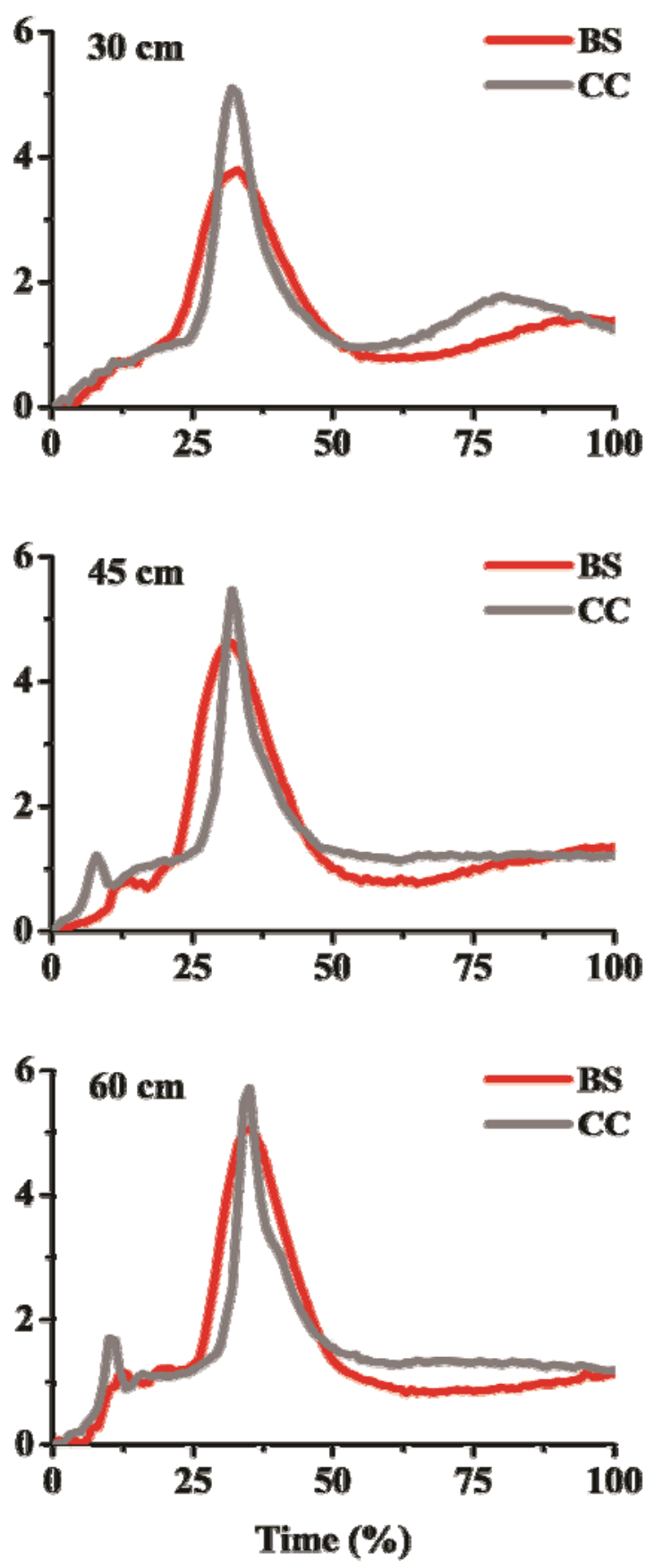

Figure 1

Representative vertical GRF-time curves in the basketball shoe (BS) and the control shoe (CC) during drop jump landing and passive landing tasks. The landing phase (time \%) was denoted by the duration of the landing between initial contact and maximum knee flexion. 
Drop Jump Landing
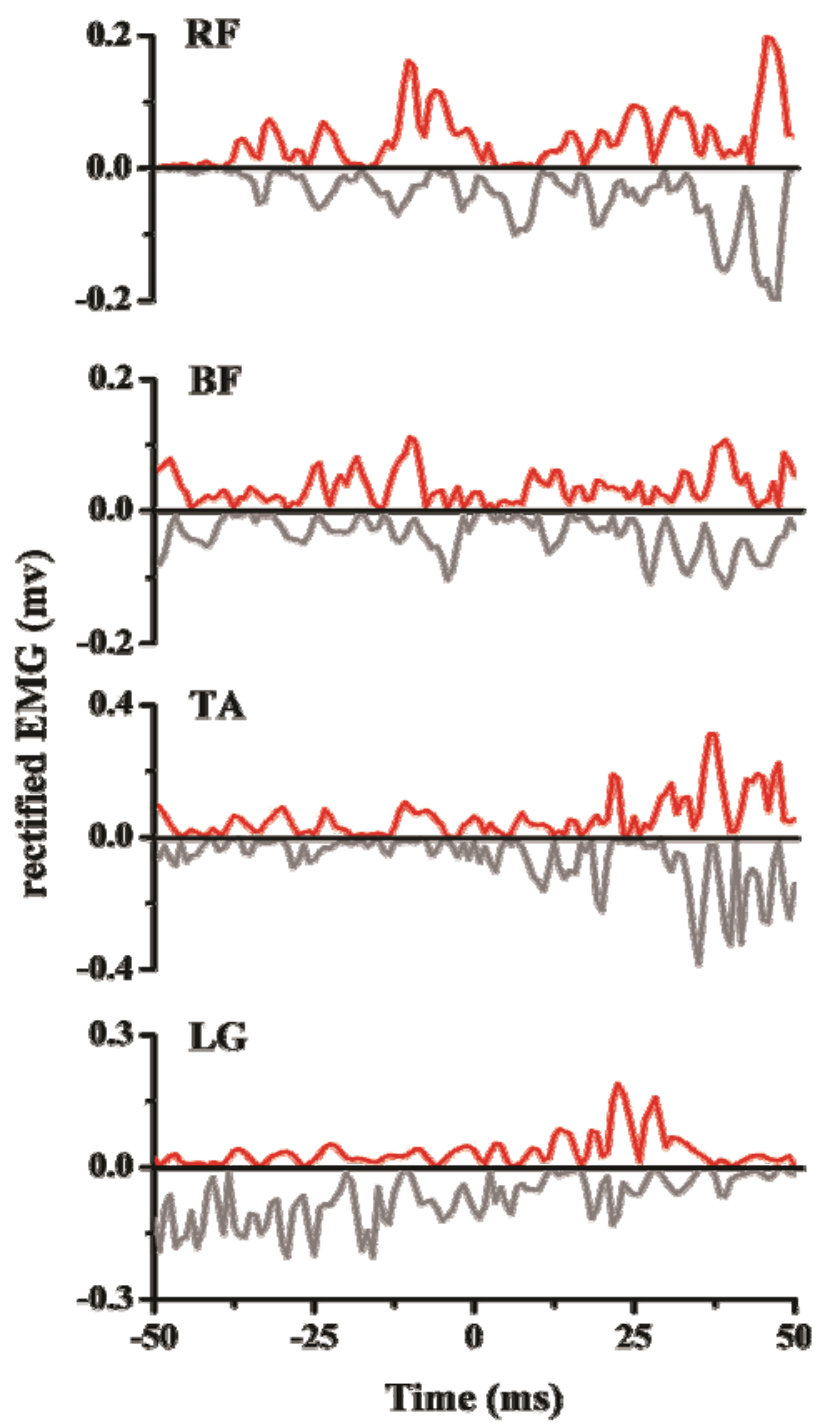

Passive Landing
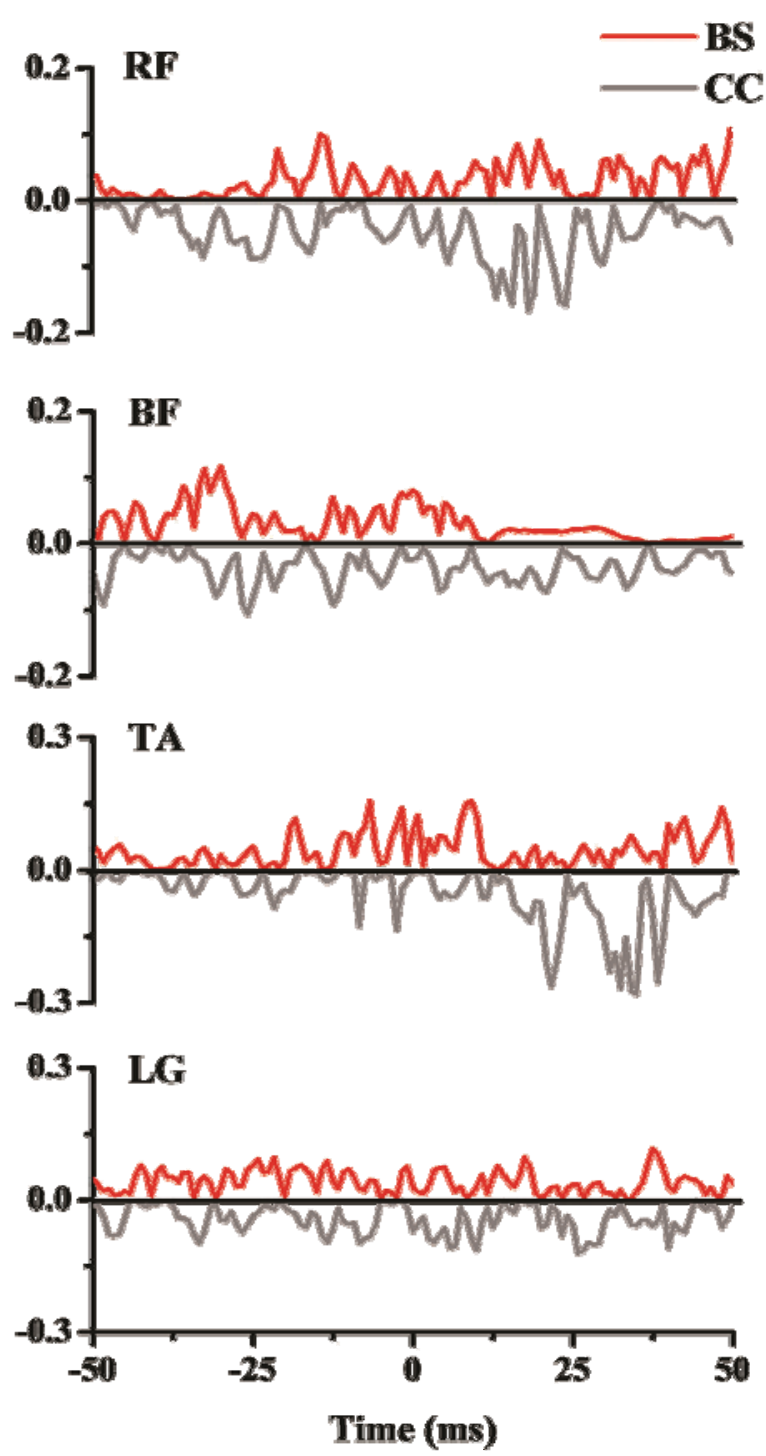

Figure 2

Representative full-wave rectified EMG curves for_the rectus femoris (RF), biceps femoris (BF), tibialis anterior (TA) and lateral gastrocnemius (LG) muscles in the basketball shoe (BS) and the control shoe (CC) during a drop jump landing and a passive landing from a $60 \mathrm{~cm}$ drop height. $0 \mathrm{~ms}$ was defined as the time of initial contact. CC data inverted to allow both curves to be visualized. 

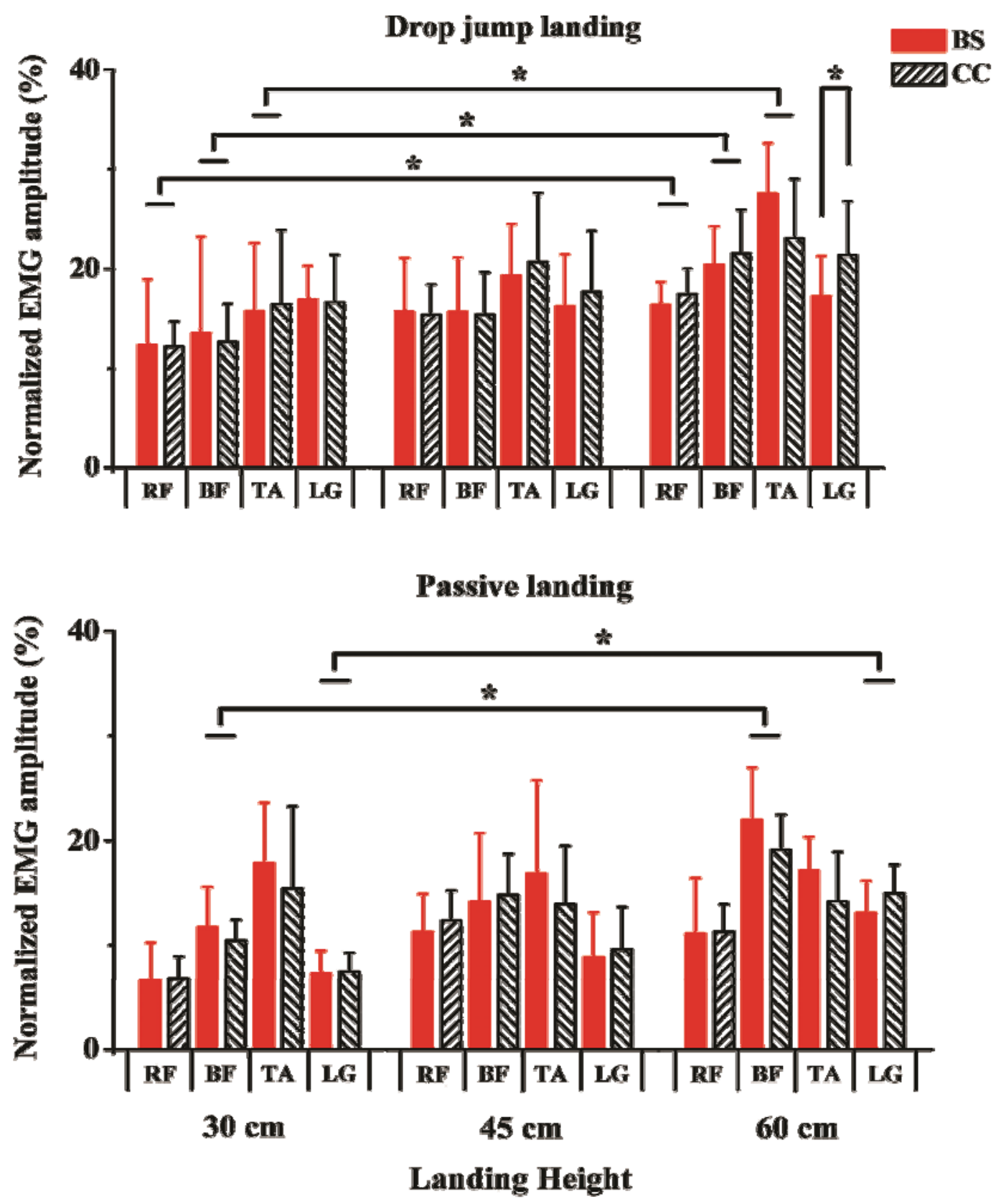

Figure 3a

Comparison of pre-activation of the rectus femoris (RF), biceps femoris (BF), tibialis anterior (TA) and lateral gastrocnemius ( $L G)$ the between basketball shoe (BS) and the control shoe (CC) in drop jump landing and passive landing tasks. 


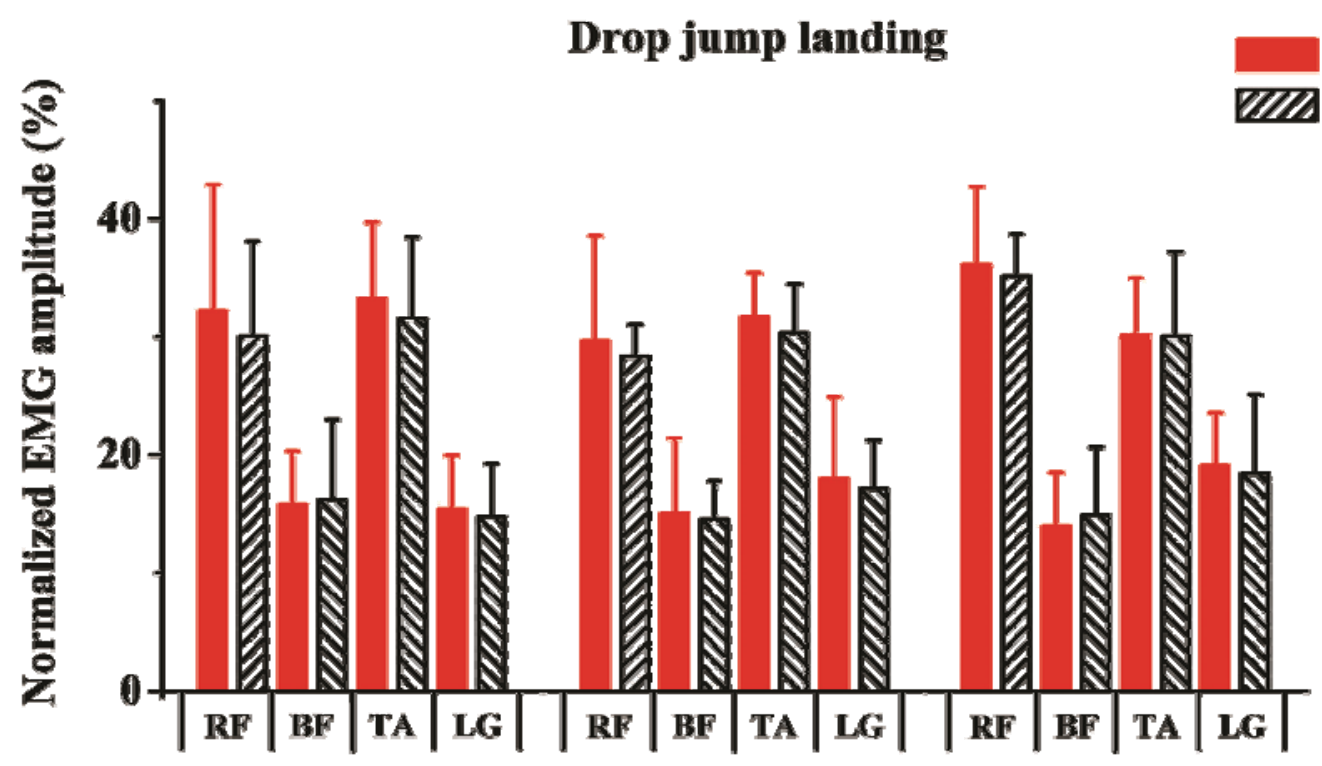

Passive landing

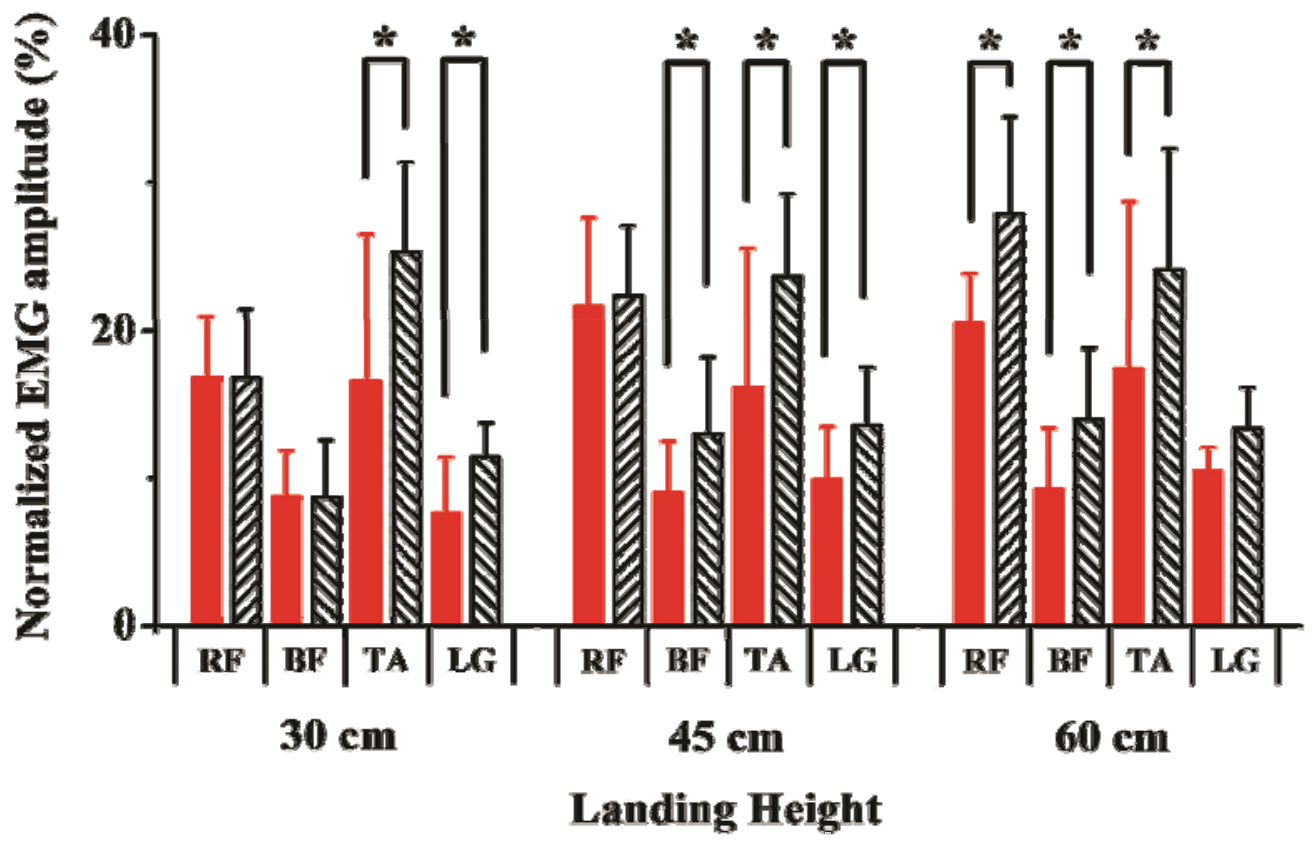

Figure $3 b$

Comparison of post-activation of the rectus femoris (RF), biceps femoris (BF), tibialis anterior (TA) and lateral gastrocnemius (LG) between the basketball shoe (BS) and the control shoe (CC) in drop jump landing and passive landing tasks. 


\section{Discussion}

This study adopted two different landing styles, an active drop jump landing (DJL) and an unexpected passive landing (PL), to assess the shoe effect on impact signals and muscle activation. During active landing, no significant difference was found between the two shoe conditions in peak vertical GRF and the peak loading rate (Table 1). Moreover, no significant differences in EMGRMS were observed for any of the tested muscles during both the pre- and postactivation phases of DJL (Figures 2 and 3).

The DJL employed in this study was a pre-programmed control landing movement; it was performed from a height onto a force plate, followed immediately by a vertical jump through anticipatory pre-activation and/or the central motor program (Horita et al., 2002; Santello and McDonagh, 1998). These findings supported the conclusion proposed by most of the impactrelated research, which revealed that the characteristics of the input signal (impact), substantially affected by the lower body mass and touchdown speed, were relatively insensitive to the alternation of the footwear cushioning property during the impact phase of the landing (Hennig et al., 1996; Milani et al., 1997). Conceptually, the time-varying vertical impact is mainly determined by the effective mass and the velocity of the body, which can be expressed as (Lieberman et al., 2010):

$$
\int_{0^{-}}^{T} F_{z}(t)=M_{\mathrm{eff}}\left(-v_{\text {foot }}+g T\right)
$$

where Meff is the effective mass determined by leg geometry (e.g. a joint angle) and lower extremity stiffness, vfoot is the vertical velocity of the foot at the landing, and $\mathrm{T}$ is the time from the touchdown to the peak impact. Clark et al. (1983) indicated no effect of a softer shoe on the peak impact forces compared with a hard shoe during the touchdown. Other studies similarly reported that the impact magnitude and the peak loading rate were relatively insensitive to changes in the hardness and/or materials of the shoe midsole at a consistent velocity (Nigg et al., 1987). This outcome may be partially attributed to human body adjustments (i.e., higher levels of muscle activation in the present study), which would appropriately occur to reduce impacts in terms of kinematic adaptation or increasing joint angles of the lower extremity (Wakeling et al., 2003). However, this possibility still needs to be confirmed and the long-term shoe (training) effects on muscle activation patterns require further investigation.

By contrast, during PL, the magnitude of both FZmax and GZmax was significantly lower in the BS condition than in the CC condition across all three heights (Table 1). Both GRF and loading rate variables showed that wearing cushioned shoes can induce greater shock attenuation compared to the control condition. Furthermore, the post-activation levels of four tested muscles (TA, LG, RF and BF) were significantly lower in BS than those of CC from at least one drop height $(p<0.05)$ (Figure $3 b)$. The findings imply that wearing high-cushioned shoes can reduce the magnitude of impact and decrease muscle post-activation if the muscles are improperly activated.

Potthast et al. (2010) reported that the hardness of the interfaces explained less than 10\% of the variance of impacts, whereas muscular activation changes explained from 35 to $48 \%$ of the variance. The authors concluded that muscle forces had considerably greater effects than interface hardness on the severity of impacts on the human body. However, muscles are not always activated properly during sports activities. Several factors, such as bad technique, fatigue and unanticipated events, may reduce muscle activation, which underlines the need for additional protection from cushioning shoes or unique structures (Boyer and Nigg, 2004).

In a pre-programmed movement, e.g., running or a landing, muscle activities shortly before and after ground contact are associated with preparing the locomotor system for and responding to the impact, and thus executing the movement task (Chumanov et al., 2012; Nigg and Wakeling, 2001). These activities are predetermined through the impact signal experienced during previous landings. The tuning function of muscle activity (muscle tuning) suggests that impact signals are sensed and the central nervous system responds by tuning, if necessary, the activation of the corresponding major muscle groups, in reducing impact loading during athletic activities (Nigg and Wakeling, 
2001). On the contrary, the participants in the current study were not aware of and did not properly prepare for the sudden drop landings. These unanticipated changes, primarily due to inadequate postural control and improper muscular activity in the lower extremity, can eliminate (or partially eliminate) the biofeedback and negatively affect the adaptation strategy of the neuromuscular system (Hardin et al., 2004). A related study (Boyer and Nigg, 2006) reported a significant decrease in the EMG activation intensity of leg muscles during landings on the unexpected surface compared to the expected surface landing. The authors proposed that the changes in muscle activity in response to different landing conditions might serve to control the softtissue vibrations of the lower extremity following the impact.

Basically, the unexpected position change, such as in PL, can basically reduce muscle involvement and cause an inadequate adaptation strategy of the neuromuscular system in response to different impacts and input signals received by the human body (Gerritsen et al., 1995; Hardin et al., 2004). Consequently, the cushioned footwear adopted in this study plays an important role, similar to those of the movement control strategies (muscle tuning) (Boyer and Nigg, 2007) used in DJLs, in reducing the magnitude of both FZmax and GZmax, reducing muscle postactivation, and preventing potential injuries. Similarly, a previous study reported that the effect of the soft impact interface on the impacts exerted a significant decrease in the peak vertical GRF compared with the unexpected hard landings (Boyer and Nigg, 2006). The issue of whether the reduction in muscle activity represents the clearly beneficial effects of wearing high-cushioned shoes remains uncertain; thus, future studies should further investigate the role of footwear in minimizing unnecessary muscular activity in endurance sports.

Assessment of lower limb kinematics (e.g., joint angles and range of motion), accompanied simultaneously by joint kinetics and muscle activation, was warranted to provide further evidence on neuromuscular patterns associated with landing tasks and interface/shoe configurations. Additionally, to avoid potential impact-induced injuries during a barefoot landing, especially in a passive drop landing, a shoe with nearly no cushioning ability was adopted in this study to simulate a barefoot landing. This unique shoe choice should be considered in the interpretation of results. Furthermore, future studies should likewise look at the effects of midsole properties (hardness and materials), and landing strategies, which are issues that this study was not designed to address.

\section{Conclusions}

In summary, wearing basketball footwear did not significantly influence peak impact force, the peak loading rate, as well as the neuromuscular activity patterns during an active landing. This result indicates that shoe condition may have limited effects on reducing the impact if neuromuscular adjustments appropriately occur during active movements (i.e., jump landings and running). However, under the condition in which the related neuromuscular system is improperly activated, a neuromuscular adaptation occurs (i.e., reduced magnitude of impact and decreased muscle post-activation) in response to different impacts (shoe/surface) and thus inputs signals to the body.

\section{Acknowledgements}

This research was supported by the NNSF of China (11302131), the Doctoral Fund of Ministry of Education of China (20123156120003), the Innovation Program of Shanghai Municipal Education Commission (14YZ125), and the Science and Technology Commission of Shanghai (14DZ1103500).

\section{References}

Agel J, Olson DE, Dick R, Arendt EA, Marshall SW, Sikka RS. Descriptive epidemiology of collegiate women's basketball injuries: National Collegiate Athletic Association Injury Surveillance System, 1988-1989 through 2003-2004. J Athl Train, 2007; 42: 202-210 
Basmajian JV, DeLuca CJ. Muscle alive: Their functions revealed by electromyography. 5th ed. Baltimore: Williams \& Wilkins; 1985

Beck TW, Stock MS, DeFreitas JM. Time-frequency analysis of surface electromyographic signals during fatiguing isokinetic muscle actions. J Strength Cond Res, 2012; 26: 1904-1914

Beynnon BD, Vacek PM, Murphy D, Alosa D, Paller D. First-time inversion ankle ligament trauma: the effects of sex, level of competition, and sport on the incidence of injury. Am J Sports Med, 2005; 33: 1485-1491

Borowski LA, Yard EE, Fields SK, Comstock RD. The epidemiology of US high school basketball injuries, 2005-2007. Am J Sports Med, 2008; 36: 2328-2335

Boyer KA, Nigg BM. Muscle activity in the leg is tuned in response to impact force characteristics. J Biomech, 2004; 37: 1583-1588

Boyer KA, Nigg BM. Muscle tuning during running: implications of an un-tuned landing. J Biomech Eng, 2006; 128: 815-822

Boyer KA, Nigg BM. Changes in muscle activity in response to different impact forces affect soft tissue compartment mechanical properties. J Biomech Eng, 2007; 129: 594-602

Brüggemann GP, Brüggemann L, Heinrich K, Müller M, Niehoff A. Biological tissue response to impact like mechanical loading. Footwear Science, 2011; 3: 13-22

Camic CL, Housh TJ, Zuniga JM, Russell Hendrix C, Bergstrom HC, Traylor DA, Schmidt RJ, Johnson GO. Electromyographic and mechanomyographic responses across repeated maximal isometric and concentric muscle actions of the leg extensors. J Electromyogr Kinesiol, 2013; 23: 342-348

Chumanov ES, Wille CM, Michalski MP, Heiderscheit BC. Changes in muscle activation patterns when running step rate is increased. Gait Posture, 2012; 36: 231-235

Clarke TE, Frederick EC, Cooper LB. Effects of shoe cushioning upon ground reaction forces in running. Int J Sports Med, 1983; 4: 247-251

Derrick TR, Dereu D, McLean SP. Impacts and kinematic adjustments during an exhaustive run. Med Sci Sports Exerc, 2002; 34: 998-1002

Frederick EC, Hagy JL. Factors affecting peak vertical ground reaction forces in running. Int $J$ of Sport Biomech, 1986; 2: 41-49

Fu W, Fang Y, Liu Y, Hou J. The effect of high-top and low-top shoes on ankle inversion kinematics and muscle activation in landing on a tilted surface. J Foot Ankle Res, 2014; 7: 14

Fu W, Liu Y, Zhang S, Xiong X, Wei S. Effects of local elastic compression on muscle strength, electromyographic, and mechanomyographic responses in the lower extremity. J Electromyogr Kinesiol, 2012; 22: 44-50

Gabriel DA, Lester SM, Lenhardt SA, Cambridge ED. Analysis of surface EMG spike shape across different levels of isometric force. J Neurosci Methods, 2007; 159: 146-152 
Gerritsen KG, van den Bogert AJ, Nigg BM. Direct dynamics simulation of the impact phase in heel-toe running. J Biomech, 1995; 28: 661-668

Gross TS, Nelson RC. The shock attenuation role of the ankle during landing from a vertical jump. Med Sci Sports Exerc, 1988; 20: 506-514

Hamill J, Bates BT, Knutzen KM, Sawhill JA. Variations in ground reaction force parameters at different running speeds. Human Movement Science, 1983; 2: 47-56

Hardin EC, van den Bogert AJ, Hamill J. Kinematic adaptations during running: effects of footwear, surface, and duration. Med Sci Sports Exerc, 2004; 36: 838-844

Hennig EM, Valiant GA, Liu Q. Biomechanical variables and the perception of cushioning for running in various types of footwear. J Appl Biomech, 1996; 12: 143-150

Horita T, Komi PV, Nicol C, Kyrolainen H. Interaction between pre-landing activities and stiffness regulation of the knee joint musculoskeletal system in the drop jump: implications to performance. Eur J Appl Physiol, 2002; 88: 76-84

Huang YC, Huang WJ, Tsai FJ, Liu Y. Neuromechanical strategy in visually impaired student during downward stepping. Journal of Mechanics in Medicine and Biology, 2005; 5: 261-265

Lafortune MA, Hennig EM, Lake MJ. Dominant role of interface over knee angle for cushioning impact loading and regulating initial leg stiffness. J Biomech, 1996; 29: 1523-1529

Lawrence RK, 3rd, Kernozek TW, Miller EJ, Torry MR, Reuteman P. Influences of hip external rotation strength on knee mechanics during single-leg drop landings in females. Clin Biomech (Bristol, Avon), 2008; 23: 806-813

Lieberman DE, Venkadesan M, Werbel WA, Daoud AI, D'Andrea S, Davis IS, Mang'eni $\mathrm{RO}$, Pitsiladis Y. Foot strike patterns and collision forces in habitually barefoot versus shod runners. Nature, 2010; 463: 531-535

McNitt-Gray JL. Kinetics of the lower extremities during drop landings from three heights. $J$ Biomech, 1993; 26: 1037-1046

Milani TL, Hennig EM, Lafortune MA. Perceptual and biomechanical variables for running in identical shoe constructions with varying midsole hardness. Clin Biomech (Bristol, Avon), 1997; 12: 294-300

Nigg BM, Bahlsen HA, Luethi SM, Stokes S. The influence of running velocity and midsole hardness on external impact forces in heel-toe running. J Biomech, 1987; 20: 951-959

Nigg BM, Wakeling JM. Impact forces and muscle tuning: a new paradigm. Exerc Sport Sci Rev, 2001; 29: 37-41

Potthast W, Bruggemann GP, Lundberg A, Arndt A. The influences of impact interface, muscle activity, and knee angle on impact forces and tibial and femoral accelerations occurring after external impacts. J Appl Biomech, 2010; 26: 1-9

Rocha-Júnior VA, Bottaro M, Pereira MC, Ferreira-Júnior JB, Carmo J, Brown LE, Nascimento 
FAO. Reliability of normalized surface electromyographic signals of maximal upper-body isokinetic strength. Isokinet Exerc Sci, 2015; 23: 1-12

Ruan M, Li L. Approach run increases preactivation and eccentric phases muscle activity during drop jumps from different drop heights. J Electromyogr Kinesiol, 2010; 20: 932-938

Santello M, McDonagh MJ. The control of timing and amplitude of EMG activity in landing movements in humans. Exp Physiol, 1998; 83: 857-874

Wakeling JM, Liphardt AM, Nigg BM. Muscle activity reduces soft-tissue resonance at heel-strike during walking. J Biomech, 2003; 36: 1761-1769

Wakeling JM, Pascual SA, Nigg BM. Altering muscle activity in the lower extremities by running with different shoes. Med Sci Sports Exerc, 2002; 34: 1529-1532

Wikstrom EA, Tillman MD, Kline KJ, Borsa PA. Gender and limb differences in dynamic postural stability during landing. Clin J Sport Med, 2006; 16: 311-315

\section{Corresponding author:}

\section{Weijie Fu, PhD}

Key Laboratory of Exercise and Health Sciences of Ministry of Education

Shanghai University of Sport

200438 Shanghai, China

Phone: +86-21-51253239; Fax: +86-21-51253242

Email: fuweijie@sus.edu.cn 